\title{
Beliefs and practices regarding electronic cigarettes in smoking cessation among healthcare professionals in Slovenia
}

\author{
Helena Koprivnikar', Tina Zupanič ${ }^{1}$, Jerneja L. Farkas ${ }^{1,2,3}$
}

\begin{abstract}
INTRODUction Electronic cigarettes (ECs) have generated extensive discussion about their role in smoking cessation. The Slovenia National Institute of Public Health's recommendations state that ECs are not to be recommended for smoking cessation or reduction. The aim of this study was to explore how healthcare professionals working in the field of preventive healthcare and smoking cessation in Slovenia communicate with and counsel patients regarding electronic cigarettes and smoking cessation or reduction.

METHODS A cross-sectional, web-based survey was conducted among healthcare professionals working in the field of preventive healthcare and smoking cessation in Slovenia. A total of 479 healthcare professionals were included in the analysis. RESULTS While a minority of participants $(12.7 \%)$ do or would recommend electronic cigarettes for smoking cessation or reduction in general, a higher proportion of participants $(33.1 \%)$ would recommend electronic cigarettes to specific groups of patients. Knowledge on electronic cigarettes was the key determinant of differences in recommendations. Only a minority of participants (9.1\%) reported availability of workplace guidelines/recommendations regarding counselling about electronic cigarettes.

CONCLUSIONS Training programmes, educational materials and existing guidelines/ recommendations regarding counselling about electronic cigarette use should be provided and distributed among healthcare professionals, together with efforts to ensure compliance to official guidelines/recommendations.
\end{abstract}

\section{AFFILIATION}

1 National Institute of Public Health, Ljubljana, Slovenia

2 General Hospital Murska Sobota, Murska Sobota, Slovenia

3 Faculty of Medicine, University of Ljubljana, Ljubljana, Slovenia

\section{CORRESPONDENCE TO}

Helena Koprivnikar. Analysis and Development of Health Department, National Institute of Public Health, Trubarjeva 2, 1000 Ljubljana, Slovenia.

E-mail: helena.koprivnikar@nijz.si

\section{KEYWORDS}

smoking cessation, counselling, healthcare professionals, electronic cigarettes, preventive health care

Received: 26 September 2019

Revised: 22 November 2019

Accepted: 3 December 2019

\section{INTRODUCTION}

Electronic cigarettes (ECs) are relatively new nontobacco products, intended for nicotine delivery to users, that have an important impact on the landscape of tobacco use, tobacco control and nicotine dependence. Their increasing use during the past years has, amongst other things, generated extensive discussion about their role in smoking cessation ${ }^{1-3}$. Supporters of ECs advocate their efficiency in smoking cessation and argue that ECs are less harmful compared to conventional cigarettes and that smokers would have health benefits if they switch from conventional cigarettes to ECs. Nevertheless, there are not enough reliable data and research to establish that ECs are effective in smoking cessation ${ }^{4,5}$. They maintain nicotine dependence, quality standards of manufacture are questionable, and effects on health from long-term use are unknown ${ }^{4}$. Not only the public but also healthcare professionals are receiving mixed messages.

Healthcare professionals are a credible and trustworthy source of health information for their patients including risks related to smoking, smoking cessation, and ECs; they are also providers of smoking cessation interventions. They are encountering increasing number of patients that are 
having experiences with and queries about $\mathrm{ECs}^{6,7}$. Healthcare professionals' advice and counselling are important in shaping patients' decisions about ECs, but currently deal with limited data on their safety and effectiveness. Literature on healthcare professionals' communication and counselling about ECs to their patients, although limited, shows that a certain proportion of healthcare professionals recommend ECs for smoking cessation, but the majority do not $^{8,9}$, which is aligned with the latest recommendations and guidelines from professional associations $^{10-14}$. The Slovenia National Institute of Public Health's recommendations state that ECs should not be recommended for smoking cessation or reduction. Healthcare professionals should recommend licensed smoking cessation treatments (nicotine replacement therapy or prescription medications) and encourage patients to use them if they are thinking about using ECs. If a patient still decides to use ECs for smoking cessation or reduction, it is recommended that healthcare professionals inform the patient on what is known about their safety and efficacy, about no benefit of dual use, and encourage the patient to stop using ECs in the nearest future.

There are data available on EC use in Slovenia among adults and youth ${ }^{15,16}$, but there is no research about healthcare professionals' attitudes, beliefs, knowledge and practices regarding ECs. The aim of this study was to explore how healthcare professionals working in the field of preventive healthcare and smoking cessation in Slovenia communicate with and counsel patients regarding ECs and smoking cessation or reduction.

\section{METHODS}

\section{Study design and respondents}

A cross-sectional, web-based survey was conducted among healthcare professionals working in the field of preventive healthcare and smoking cessation in Slovenia, which included healthcare professionals working in the Family Medicine Practices, Community Care, and Health Education Centres/Health Promotion Centres, in the Community Healthcare Centres of Slovenia, as well as counsellors on Quitline, and those working in Outpatient Cardiac Rehabilitation Units. These groups of healthcare professionals were our sample frame as they have the key role in prevention and cessation of smoking.

The invitation to participate in the survey, including the link to the survey, was sent to either the direct public e-mails of healthcare professionals or contact persons with the request that they forward the invitation to their co-workers; altogether 1122 invitations were sent.

The web survey was open between November 2018 and March 2019. Reminders were sent to increase the response rate. Participation was anonymous. The study protocol was evaluated and approved by the Republic of Slovenia National Medical Ethics Committee (Approval No.0120$460 / 2018 / 4)$.

\section{Questionnaire and survey measures}

The questionnaire was developed by two public health specialists and a statistician, based on previous similar published research ${ }^{17-21}$, and was carefully reviewed for terminology and language. The questionnaire was piloted in a small group of healthcare professionals. The final questionnaire consisted of 59 questions (53 closed questions and 6 open questions) from different domains, including respondents' characteristics, counselling patients about ECs, availability of guidelines/recommendations at work, and perceived and actual knowledge about ECs.

\section{Characteristics of respondents}

Respondents were asked to report: gender, year of birth, education, provider type, workplace, statistical region of the workplace, number of years at current workplace, number of years working with patients, usual number of patients per week, age group of the patients, and whether they provide smoking cessation support and counselling to their patients. Respondents were asked whether they smoke or have ever smoked tobacco, including manufactured cigarettes, rollyour-own cigarettes, cigars, cigarillos, waterpipe, but not ECs or heat-not-burn tobacco products (HNB); with answers ranging from daily smoking, occasional smoking, smoking in the past, tried in the past, and never smoked. We recoded answers into three groups: current smokers, ex-smokers, and never smokers (including experimenters). Number of cigarettes smoked on average per day and number of years of smoking were used to calculate pack years of cigarette smoking. Respondents were asked whether they have 
ever used or currently use ECs, HNB or smokeless tobacco. Use of these products (referred to as other products in what follows) was assessed by a question on use and frequency of use, with the same response options provided as in the question about tobacco smoking. The answers were dichotomised to never users and ever users of at least one other product.

\section{Counselling patients about ECs}

Respondents were first asked to report how they counsel or would counsel patients if asked about efficacy of ECs in smoking cessation or reducing the number of cigarettes smoked, with answers ranging from: I recommend ECs, as they can be effective in this respect; I would not recommend ECs for this purpose, but the decision is up to you; Do not use ECs for this purpose; There are not enough data and research available to advise you or answer your questions; I do not know enough about ECs to advise you or answer your questions; and Other. Respondents were then asked if they recommend or would recommend ECs to their patients for smoking cessation or reduction of number of cigarettes smoked (yes, proactively; yes, but only if a patient asks me about this; no). We dichotomised answers into two groups, those that recommend ECs and those that do not. Finally, they were asked to which groups of patients, if at all, they would recommend ECs (those that already use ECs; those that want to reduce the number of cigarettes smoked; those that want to reduce the number of cigarettes smoked before they stop smoking; those that want to use ECs in places where smoking is banned or places where they should not smoke; those that want to stop smoking; those that want to stop smoking but were unsuccessful in the past, although they tried different types of official smoking cessation help; those that decline other official smoking cessation help; the more addicted smokers; no one; other).

\section{Availability of guidelines/recommendations at work}

Respondents were asked if there are any guidelines/ recommendations for their communication with patients about ECs at their workplace (yes; no; I don't know). If a respondent reported that guidelines/ recommendations existed, they were asked about the content of these guidelines/recommendations with the possibility to choose among a list of pre-defined answers (multiple-choice question).

\section{Knowledge about ECs}

Respondents had to identify which of the stated ingredients are present in EC liquid or aerosol and rate a number of statements as correct or incorrect. We created a knowledge score from both questions. For each correct answer the respondent received 1 point, for incorrect -1 , for 'I don't know' 0 points, with the score ranging from -33 to +33 . We calculated a knowledge score for all respondents that answered at least two-thirds of the questions.

\section{Statistical analysis}

All together we received 523 at least partially completed surveys. Surveys from 44 respondents that did not work with patients and/or did not answer the majority of questions in the survey were excluded, and thus surveys from 479 respondents were included in the analysis. Missing data were excluded from the analysis. Descriptive statistics were calculated for all questions. In many questions, response categories were grouped into lower number of categories, as described previously, to ensure an adequate sample size within each category. We used chi-squared $(\times 2)$ test to examine the association between selected variables. Multivariate logistic regression analyses were performed to estimate odds ratios (ORs) and their $95 \%$ confidence intervals (95\% CIs) with twosided probability $\mathrm{p}$-values. We included only variables that were shown to have statistically significant associations in bivariate analyses. In all cases a p-value $<0.05$ was considered as statistically significant. All analyses were conducted in IBM SPSS ver. 25 (SPSS Inc., Chicago, IL, USA).

\section{RESULTS}

The characteristics of the respondents included in the analysis are shown in Table 1.

Among respondents who were or were going to be asked by their patients about the effectiveness of ECs in smoking cessation or reducing the number of cigarettes smoked, the majority felt that they would not be able to give advice or recommend ECs as being effective. Responses were found to differentiate significantly by a number of variables, as shown in Tables 2 and 3 . The majority $(21 / 27$ or $77.7 \%)$ of those that recommend or 
Table 1. Characteristics of the respondents in the study

\begin{tabular}{|c|c|c|c|}
\hline Characteristics & Calcgories & n & $\%$ \\
\hline Total & & 479 & 100.0 \\
\hline \multirow[t]{2}{*}{ Gender } & Male & 47 & 9.8 \\
\hline & Female & 431 & 90.2 \\
\hline \multirow[t]{2}{*}{ Age (years) } & 20-39 & 235 & 49.1 \\
\hline & $\geq 40$ & 244 & 50.9 \\
\hline \multirow[t]{2}{*}{ Education } & $\begin{array}{l}\text { Higher vocational, short-term } \\
\text { higher or less }\end{array}$ & 86 & 18.0 \\
\hline & Professional higher or more & 392 & 82.0 \\
\hline \multirow[t]{3}{*}{ Provider type } & Nurse or midwife & 356 & 74.5 \\
\hline & Physician & 70 & 14.6 \\
\hline & Other & 52 & 10.9 \\
\hline \multirow[t]{6}{*}{ Workplace } & $\begin{array}{l}\text { Health Education Centres/ } \\
\text { Health Promotion Centres }\end{array}$ & 122 & 25.6 \\
\hline & Quitline telephone & 10 & 2.1 \\
\hline & Family Medicine Practices & 221 & 46.3 \\
\hline & Community care & 93 & 19.5 \\
\hline & $\begin{array}{l}\text { Outpatient Cardiac } \\
\text { Rehabilitation Units }\end{array}$ & 14 & 2.9 \\
\hline & Other & 17 & 3.6 \\
\hline \multirow[t]{2}{*}{ Cohesion region } & Eastern Slovenia & 254 & 53.0 \\
\hline & Western Slovenia & 225 & 47.0 \\
\hline \multirow{3}{*}{$\begin{array}{l}\text { Number of } \\
\text { years at current } \\
\text { workplace }\end{array}$} & $<1$ & 96 & 20.1 \\
\hline & $1-9$ & 223 & 46.8 \\
\hline & $\geq 10$ & 158 & 33.1 \\
\hline \multirow{3}{*}{$\begin{array}{l}\text { Number of years } \\
\text { working with } \\
\text { patients }\end{array}$} & $<1$ & 65 & 13.6 \\
\hline & $1-9$ & 156 & 32.7 \\
\hline & $\geq 10$ & 256 & 53.7 \\
\hline \multirow{3}{*}{$\begin{array}{l}\text { Usual number of } \\
\text { patients/week }\end{array}$} & $<20$ & 135 & 29.5 \\
\hline & $20-40$ & 169 & 36.9 \\
\hline & $\geq 41$ & 154 & 33.6 \\
\hline \multirow{2}{*}{$\begin{array}{l}\text { Patients' age } \\
\text { group }\end{array}$} & Only adults $\geq 26$ years & 317 & 66.9 \\
\hline & Others & 157 & 33.1 \\
\hline \multirow{2}{*}{$\begin{array}{l}\text { Provision } \\
\text { of smoking } \\
\text { cessation } \\
\text { support }\end{array}$} & Yes & 378 & 79.6 \\
\hline & No & 97 & 20.4 \\
\hline \multirow[t]{5}{*}{ Smoking status } & Never smoker & 223 & 46.7 \\
\hline & $\begin{array}{l}\text { Smoked on few occasions in } \\
\text { life }\end{array}$ & 123 & 25.7 \\
\hline & Ex-smoker & 78 & 16.3 \\
\hline & Current occasional smoker & 25 & 5.2 \\
\hline & Current daily smoker & 29 & 6.1 \\
\hline \multirow[t]{2}{*}{ Ever heard of EC } & Yes & 469 & 99.4 \\
\hline & No & 3 & 0.6 \\
\hline \multirow[t]{3}{*}{ EC use } & Current user & 8 & 1.7 \\
\hline & Ex-user and experimenter & 25 & 5.4 \\
\hline & Never user & 431 & 92.9 \\
\hline \multirow{2}{*}{$\begin{array}{l}\text { Ever use of other } \\
\text { products (EC, } \\
\text { HNB, smokeless) }\end{array}$} & Yes & 54 & 21.5 \\
\hline & No & 197 & 78.5 \\
\hline
\end{tabular}

would recommend ECs for smoking cessation also recommend or would recommend EGs for reduction of the number of cigarettes smoked.

When asked more directly about whether they recommend or would recommend ECs for smoking cessation or reducing the number of cigarettes smoked, a minority responded positively. The majority of those that recommend or would recommend ECs do no report proactive recommendations, but do so mostly as a result of patients' queries. For smoking cessation, 9.9\% do or would recommend ECs only if a patient asks and $2.8 \%$ proactively; for reducing the number of cigarettes smoked, $11.8 \%$ do or would recommend ECs only if a patient asks and $2.1 \%$ proactively. Again, the majority (49/59 or $90.7 \%$ ) of those that recommend or would recommend ECs for smoking cessation, also recommend or would recommend ECs for reduction of number of cigarettes smoked. Responses of those that recommend ECs for smoking cessation or cutting down were found to differ significantly from those that do not recommend ECs by different variables, as shown by the results of bivariate and multivariate analyses (Table 4). We also more closely explored the group of ex-smokers that recommend ECs for either purpose. We found that all ex-smokers that are current EC users recommend ECs, as does the vast majority of ex-smokers that used or experimented with ECs in the past.

When respondents were asked to which groups of patients, if at all, they would recommend ECs, again the majority responded that they would not recommend ECs to any of their patients (66.9\%). But $12.7 \%(n=53)$ would recommend ECs to patients that want to stop smoking and were unsuccessful in the past, although they tried different types of official smoking cessation help, $11.0 \%(n=46)$ to patients that decline other types of official smoking cessation help and the same number and per cent to patients that want to reduce the number of cigarettes smoked before they stop smoking, $9.4 \%(n=39)$ to patients that want to stop smoking, $7.7 \%(n=32)$ to patients that want to reduce number of cigarettes smoked, $5.3 \%(\mathrm{n}=22)$ to heavily dependent patients, $5.0 \%$ $(\mathrm{n}=53)$ to patients that already use ECs and $2.2 \%$ $(n=9)$ to patients that want to use ECs in places where smoking is banned or places where they should not smoke, even though in Slovenia EC use 
Table 2. Respondents' counselling $(\mathrm{C1}-\mathrm{C} 5)^{*}$ on patient's queries about EC effectiveness in smoking cessation (only significant results are shown)

\begin{tabular}{|c|c|c|c|c|c|c|c|c|c|c|c|}
\hline & \multicolumn{2}{|c|}{$\mathrm{CI}$} & \multicolumn{2}{|c|}{$\mathrm{C} 2$} & \multicolumn{2}{|c|}{ C3 } & \multicolumn{2}{|c|}{$C 1$} & \multicolumn{2}{|c|}{ C5 } & \multirow[b]{2}{*}{ p } \\
\hline & n & $\%$ & n & $\%$ & n & $\%$ & n & $\%$ & n & $\%$ & \\
\hline Total & 141 & 33.8 & 94 & 22.5 & 90 & 21.6 & 64 & 15.3 & 27 & 6.4 & \\
\hline \multicolumn{12}{|l|}{ Gender } \\
\hline Male & 14 & 32.6 & 7 & 16.3 & 7 & 16.3 & 6 & 14.0 & 9 & 20.9 & 0.002 \\
\hline Female & 127 & 34.1 & 87 & 23.4 & 82 & 22.0 & 58 & 15.6 & 18 & 4.8 & \\
\hline \multicolumn{12}{|l|}{ Education } \\
\hline Higher vocational, short-term higher or less & 27 & 40.9 & 10 & 15.2 & 8 & 12.1 & 13 & 19.7 & 8 & 12.1 & 0.025 \\
\hline Professional higher or more & 114 & 32.6 & 84 & 24.0 & 82 & 23.4 & 51 & 14.6 & 19 & 5.4 & \\
\hline \multicolumn{12}{|l|}{ Workplace } \\
\hline $\begin{array}{l}\text { Health Education Centres/Health Promotion } \\
\text { Centres }\end{array}$ & 22 & 19.6 & 31 & 27.7 & 34 & 30.4 & 20 & 17.9 & 5 & 4.5 & 0.004 \\
\hline Family Medicine Practices & 70 & 36.5 & 40 & 20.8 & 36 & 18.8 & 28 & 14.6 & 18 & 9.4 & \\
\hline Community care & 39 & 50.6 & 13 & 16.9 & 11 & 14.3 & 11 & 14.3 & 3 & 3.9 & \\
\hline Other & 10 & 29.4 & 10 & 29.4 & 8 & 23.5 & 5 & 14.7 & 1 & 2.9 & \\
\hline \multicolumn{12}{|l|}{ Provide smoking cessation support } \\
\hline Yes & 91 & 26.9 & 83 & 24.6 & 83 & 24.6 & 55 & 16.3 & 26 & 7.7 & $<0.001$ \\
\hline No & 49 & 65.3 & 10 & 13.3 & 7 & 9.3 & 8 & 10.7 & 1 & 1.3 & \\
\hline \multicolumn{12}{|l|}{ Ever use of other products } \\
\hline Yes & 10 & 22.2 & 10 & 22.2 & 6 & 13.3 & 9 & 20.0 & 10 & 22.2 & 0.003 \\
\hline No & 49 & 27.5 & 48 & 27.0 & 46 & 25.8 & 26 & 14.6 & 9 & 5.1 & \\
\hline \multicolumn{12}{|l|}{ Perceived knowledge } \\
\hline Very good or good & 1 & 2.2 & 12 & 26.7 & 14 & 31.1 & 8 & 17.8 & 10 & 22.2 & $<0.001$ \\
\hline Neither good, neither bad & 8 & 7.0 & 44 & 38.6 & 39 & 34.2 & 19 & 16.7 & 4 & 3.5 & \\
\hline Bad or very bad & 131 & 51.4 & 37 & 14.5 & 37 & 14.5 & 37 & 14.5 & 13 & 5.1 & \\
\hline \multicolumn{12}{|l|}{ Actual knowledge } \\
\hline Average score & 4.66 & & 13.36 & & 14.88 & & 10.79 & & 6.31 & & $<0.001$ \\
\hline \multicolumn{12}{|l|}{ Pack years of cigarette smoking } \\
\hline Average & 6.68 & & 8.35 & & 4.99 & & 8.30 & & 18.52 & & 0.008 \\
\hline
\end{tabular}

${ }^{*} \mathrm{C} 1$ : I do not know enough about EC to be able to advise you or answer your questions. C2: I would not recommend ECs for reducing the number of cigarettes smoked, but the decision is up to you. C3: Do not use ECs for reducing the number of cigarettes smoked. C4: There is not enough research and data available to advise you or answer your questions. $\mathrm{C} 5$ : I recommend ECs, as they can be effective for reducing the number of cigarettes smoked.

is banned in all enclosed public and working places and also in all vehicles with a minor present. There were some answers under 'Other' $(3.6 \%, \mathrm{n}=15)$, where respondents mainly noted they do not have enough knowledge on the matter. As this was a multiple-choice question, responses do not add up to $100 \%$.

Only a minority $(9.1 \%)$ reported availability of guidelines/recommendations on how they should advise and counsel patients on ECs at their workplace. Availability of guidelines differed by majority of professional characteristics of the provider and the workplace, as shown in Table 5, but not by region and patients' age group. The proportion of those recommending ECs for either smoking cessation, or reducing the number of cigarettes smoked, did not differ significantly by availability of workplace guidelines/recommendations, in comparison to respondents that do not recommend ECs for either purpose.

Out of 29 respondents that reported availability of guidelines or recommendations, 28 reported 
Table 3. Respondents' counselling (C1-C5)* on patient's queries about EC effectiveness in reducing the number of cigarettes smoked (only significant results are shown)

\begin{tabular}{|c|c|c|c|c|c|c|c|c|c|c|c|}
\hline & ( & 1 & C & & C & 3 & c & 1 & ( & & \\
\hline & n & $\%$ & n & $\%$ & n & $\%$ & n & $\%$ & n & $\%$ & p \\
\hline Total & 144 & 34.5 & 104 & 24.9 & 93 & 22.3 & 50 & 11.9 & 26 & 6.2 & \\
\hline Education & & & & & & & & & & & \\
\hline Higher vocational, Short-term higher or less & 28 & 41.8 & 10 & 14.9 & 10 & 14.9 & 11 & 16.4 & 8 & 11.9 & 0.019 \\
\hline Professional higher or more & 116 & 33.1 & 94 & 26.9 & 83 & 23.7 & 39 & 11.1 & 18 & 5.1 & \\
\hline Workplace & & & & & & & & & & & \\
\hline $\begin{array}{l}\text { Health Education Centres/Health Promotion } \\
\text { Centres }\end{array}$ & 25 & 22.3 & 36 & 32.1 & 35 & 31.3 & 12 & 10.7 & 4 & 3.6 & 0.001 \\
\hline Family Medicine Practices & 72 & 37.3 & 40 & 20.7 & 37 & 19.2 & 24 & 12.4 & 20 & 10.4 & \\
\hline Community care & 37 & 48.1 & 16 & 20.8 & 12 & 15.6 & 10 & 13.0 & 2 & 2.6 & \\
\hline Other & 10 & 29.4 & 12 & 35.3 & 8 & 23.5 & 4 & 11.8 & 0 & 0.0 & \\
\hline Age groups of patients & & & & & & & & & & & \\
\hline Only adults & 87 & 31.0 & 69 & 24.6 & 73 & 26.0 & 33 & 11.7 & 19 & 6.8 & 0.048 \\
\hline Also other beside adults & 57 & 42.9 & 34 & 25.6 & 19 & 14.3 & 16 & 12.0 & 7 & 5.3 & \\
\hline Provide smoking cessation support & & & & & & & & & & & \\
\hline Yes & 95 & 28.0 & 94 & 27.7 & 83 & 24.5 & 43 & 12.7 & 24 & 7.1 & $<0.001$ \\
\hline No & 48 & 64.0 & 9 & 12.0 & 10 & 13.3 & 6 & 8.0 & 2 & 2.7 & \\
\hline Ever use of other products & & & & & & & & & & & \\
\hline Yes & 12 & 26.7 & 11 & 24.4 & 7 & 15.6 & 5 & 11.1 & 10 & 22.2 & 0.006 \\
\hline No & 49 & 27.4 & 54 & 30.2 & 45 & 25.1 & 22 & 12.3 & 9 & 5.0 & \\
\hline Perceived knowledge & & & & & & & & & & & \\
\hline Very good or good & 1 & 2.2 & 12 & 26.7 & 14 & 31.1 & 7 & 15.6 & 11 & 24.4 & $<0.001$ \\
\hline Neither good, neither bad & 8 & 7.0 & 51 & 44.3 & 37 & 32.2 & 15 & 13.0 & 4 & 3.5 & \\
\hline Bad or very bad & 133 & 52.4 & 40 & 15.7 & 42 & 16.5 & 28 & 11.0 & 11 & 4.3 & \\
\hline Actual knowledge & & & & & & & & & & & \\
\hline Average score & 4.47 & & 14.32 & & 14.41 & & 9.92 & & 7.06 & & $<0.001$ \\
\hline
\end{tabular}

${ }^{*} \mathrm{C} 1$ : I do not know enough about ECs to be able to advise you or answer your questions. C2: I would not recommend ECs for reducing the number of cigarettes smoked, but the decision is up to you. C3: Do not use ECs for reducing the number of cigarettes smoked. C4: There is not enough research and data available to advise you or answer your questions. C5: I recommend ECs, as they can be effective for reducing the number of cigarettes smoked.

Table 4. Results of bivariate and multivariate analysis regarding recommending ECs for smoking cessation and reduction

\begin{tabular}{|c|c|c|c|c|c|c|c|c|}
\hline & \multicolumn{4}{|c|}{ Recommending ECs for smoking cessation } & \multicolumn{4}{|c|}{$\begin{array}{c}\text { Recommending ECs for reducing the number } \\
\text { of cigarettes smoked }\end{array}$} \\
\hline & $n$ & $\%$ & $p$ & $\begin{array}{c}\text { Results of } \\
\text { multivariate } \\
\text { logistic regression }\end{array}$ & n & $\%$ & $p$ & $\begin{array}{c}\text { Results of } \\
\text { mullivariate } \\
\text { logistic regression }\end{array}$ \\
\hline Total & $54 / 424$ & 12.7 & & & $59 / 425$ & 13.9 & & \\
\hline \multicolumn{9}{|l|}{ Gender } \\
\hline Male & $11 / 42$ & 26.2 & 0.006 & $\begin{array}{l}1.74(0.27-11.38) \\
\quad p=0.561\end{array}$ & $13 / 42$ & 31.0 & 0.001 & $\begin{array}{l}6.35(1.37-29.44) \\
\quad p=0.018\end{array}$ \\
\hline Female & $43 / 381$ & 11.3 & & 1.00 & $46 / 382$ & 12.0 & & 1.00 \\
\hline
\end{tabular}


Table 4. Continued

Recommending ECs for smoking cessation Recommending ECs for reducing the number of cigarettes smoked

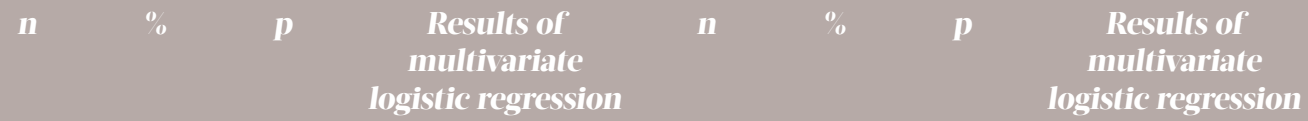

\section{Education}

\begin{tabular}{|c|c|c|c|c|c|c|c|c|}
\hline $\begin{array}{l}\text { Higher vocational, short- } \\
\text { term higher or less }\end{array}$ & $15 / 68$ & 22.1 & 0.012 & $\begin{array}{c}0.47(0.05-4.06) \\
p=0.494\end{array}$ & $16 / 69$ & 23.2 & 0.015 & $\begin{array}{c}0.47(0.07-3.46) \\
\quad p=0.462\end{array}$ \\
\hline $\begin{array}{l}\text { Professional higher or } \\
\text { more }\end{array}$ & $39 / 356$ & 11.0 & & 1.00 & $43 / 356$ & 12.1 & & 1.00 \\
\hline \multicolumn{9}{|l|}{ Smoking status } \\
\hline Current smoker & $5 / 49$ & 10.2 & & $\begin{array}{c}0.16(0.01-3.29) \\
p=0.236\end{array}$ & $7 / 49$ & 14.3 & & $\begin{array}{c}0.39(0.04-3.97) \\
p=0.426\end{array}$ \\
\hline Ex-smoker & $16 / 72$ & 22.2 & 0.030 & $\begin{array}{c}0.45(0.05-3.76) \\
p=0.459\end{array}$ & $17 / 72$ & 23.6 & 0.028 & $\begin{array}{c}0.47(0.07-3.08) \\
p=0.430\end{array}$ \\
\hline $\begin{array}{l}\text { Never smoker or smoked } \\
\text { on few occasions in life }\end{array}$ & $33 / 303$ & 10.9 & & 1.00 & $35 / 304$ & 11.5 & & 1.00 \\
\hline \multicolumn{9}{|l|}{$\begin{array}{l}\text { Ever use of other } \\
\text { products }\end{array}$} \\
\hline Yes & $16 / 46$ & 34.8 & $<0.001$ & $\begin{array}{c}2.84(0.73-11.06) \\
p=0.093\end{array}$ & $19 / 46$ & 41.3 & $<0.001$ & 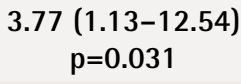 \\
\hline No & $21 / 181$ & 11.6 & & 1.00 & $21 / 181$ & 11.6 & & 1.00 \\
\hline \multicolumn{9}{|l|}{ Perceived knowledge } \\
\hline Very good or good & $14 / 46$ & 30.4 & $<0.001$ & $\begin{array}{c}3.87(0.80-18.76) \\
p=0.093\end{array}$ & $14 / 46$ & 30.4 & 0.002 & $\begin{array}{c}2.46(0.56-10.78) \\
p=0.232\end{array}$ \\
\hline Neither good, neither bad & $11 / 118$ & 9.3 & & 1.00 & $12 / 118$ & 10.2 & & 1.00 \\
\hline Bad or very bad & $27 / 258$ & 10.5 & & $\begin{array}{c}0.29(0.07-1.31) \\
p=0.108\end{array}$ & $31 / 259$ & 12.0 & & $\begin{array}{c}0.35(0.09-1.41) \\
p=0.139\end{array}$ \\
\hline \multicolumn{9}{|l|}{ Actual knowledge } \\
\hline Average score ${ }^{a}$ & $\begin{array}{l}4.94 \\
\text { (yes) }\end{array}$ & $\begin{array}{l}10.79 \\
\text { (no) }\end{array}$ & $<0.001$ & $\begin{array}{c}0.88(0.81-0.96) \\
p=0.003\end{array}$ & $\begin{array}{l}6.71 \\
\text { (yes) }\end{array}$ & $\begin{array}{l}10.62 \\
\text { (no) }\end{array}$ & 0.012 & $\begin{array}{c}0.91(0.85-0.99) \\
p=0.019\end{array}$ \\
\hline \multicolumn{9}{|l|}{$\begin{array}{l}\text { Pack years smoking } \\
\text { among current and } \\
\text { former smokers }^{b}\end{array}$} \\
\hline Average $^{a}$ & $\begin{array}{l}19.06 \\
\text { (yes) }\end{array}$ & $\begin{array}{l}6.07 \\
\text { (no) }\end{array}$ & $<0.001$ & $\begin{array}{c}1.15(0.99-1.33) \\
p=0.067\end{array}$ & $\begin{array}{l}17.50 \\
\text { (yes) }\end{array}$ & $\begin{array}{l}6.17 \\
\text { (no) }\end{array}$ & $<0.001$ & $\begin{array}{c}1.10(0.97-1.25) \\
p=0.138\end{array}$ \\
\hline
\end{tabular}

a Continuous variable. Multivariate logistic regression analyses regarding recommending ECs in cutting down the number of cigarettes smoked: $n=163$; Hosmer and Lemeshow test $\mathrm{p}=0.812$; Nagelkerke R2=0.375. $\mathrm{b}$ Never smokers and experimenters were assigned zero.

their content. The majority $(n=23)$ reported they are closest to 'We do not recommend ECs for smoking cessation, because of the lack of sufficient data on their long-term safety for health and their effectiveness in smoking cessation. We recommend well-known safe and effective types of help in smoking cessation, beside counselling and group programs, also nicotine treatment or prescription drugs'. Nineteen respondents chose 'If a patient has decided to use EC smoking cessation, and insists on it, we inform him/her about current knowledge about ECs and we encourage him/her to stop using ECs in the future. The final goal is to completely stop using any nicotine product'. Two respondents chose 'ECs are recommended for smoking cessation', one 'ECs are recommended for reduction of the number of cigarettes smoked', and one 'We support smokers if they decide to use ECs and if users experience 
Table 5. Availability of workplace guidelines recommendations on advising and counselling patients regarding ECs

\begin{tabular}{|c|c|c|c|c|c|c|c|}
\hline & \multicolumn{2}{|c|}{ Available } & \multicolumn{2}{|c|}{$\begin{array}{l}\text { Not } \\
\text { available }\end{array}$} & \multicolumn{2}{|c|}{$\begin{array}{l}\text { Don't } \\
\text { know }\end{array}$} & \multirow[b]{2}{*}{$p$} \\
\hline & n & $\%$ & $n$ & $\%$ & n & $\%$ & \\
\hline Total & 29 & 9.1 & 189 & 59.4 & 100 & 31.4 & \\
\hline \multicolumn{8}{|l|}{ Provider type } \\
\hline Nurse or midwife & 17 & 7.2 & 145 & 61.7 & 73 & 31.1 & $<0.001^{\mathrm{a}}$ \\
\hline Physician & 4 & 8.7 & 34 & 73.9 & 8 & 17.4 & \\
\hline Other & 8 & 21.6 & 10 & 27.0 & 19 & 51.4 & \\
\hline \multicolumn{8}{|l|}{ Workplace } \\
\hline $\begin{array}{l}\text { Health Education } \\
\text { Centres/Health } \\
\text { Promotion } \\
\text { Centres }\end{array}$ & 12 & 12.8 & 54 & 57.4 & 28 & 29.8 & $<0.001$ \\
\hline $\begin{array}{l}\text { Family Medicine } \\
\text { Practices }\end{array}$ & 5 & 3.7 & 94 & 69.6 & 36 & 26.7 & \\
\hline Community care & 2 & 3.4 & 29 & 50.0 & 27 & 46.6 & \\
\hline Other & 10 & 33.3 & 11 & 36.7 & 9 & 30.0 & \\
\hline \multicolumn{8}{|l|}{$\begin{array}{l}\text { Years at current } \\
\text { job }\end{array}$} \\
\hline$<1$ & 10 & 15.9 & 23 & 36.5 & 30 & 47.6 & $<0.001$ \\
\hline $1-9$ & 12 & 7.7 & 98 & 62.8 & 46 & 29.5 & \\
\hline$\geq 10$ & 7 & 7.1 & 68 & 68.7 & 24 & 24.2 & \\
\hline \multicolumn{8}{|l|}{$\begin{array}{l}\text { Years working } \\
\text { with patients }\end{array}$} \\
\hline$<1$ & 7 & 17.9 & 12 & 30.8 & 20 & 51.3 & $<0.001$ \\
\hline $1-9$ & 12 & 10.9 & 66 & 60.0 & 32 & 29.1 & \\
\hline$\geq 10$ & 10 & 5.9 & 111 & 65.7 & 48 & 28.4 & \\
\hline \multicolumn{8}{|l|}{$\begin{array}{l}\text { Number of } \\
\text { patients }\end{array}$} \\
\hline$<20$ & 17 & 18.9 & 47 & 52.2 & 26 & 28.9 & $<0.001$ \\
\hline $20-40$ & 6 & 5.2 & 65 & 56.5 & 44 & 38.3 & \\
\hline$\geq 41$ & 5 & 4.8 & 71 & 67.6 & 29 & 27.6 & \\
\hline \multicolumn{8}{|l|}{$\begin{array}{l}\text { Provide smoking } \\
\text { cessation support }\end{array}$} \\
\hline Yes & 26 & 10.0 & 162 & 62.1 & 73 & 28.0 & 0.011 \\
\hline No & 3 & 5.4 & 26 & 46.4 & 27 & 48.2 & \\
\hline
\end{tabular}

anticipated effects'. Respondents did not specify other types of guidelines or recommendations with response 'Other'.

\section{DISCUSSION}

The majority of respondents in our study do not recommend or would not recommend ECs to their patients for smoking cessation or reducing the number of cigarettes smoked, consistent with other studies ${ }^{8,9}$ and aligned with current recommendations in Slovenia. However, responses of those recommending ECs in other studies substantially differ, from none to a third or more ${ }^{7,9,18-20,22-24}$. Differences in responses can be attributed to the actual diversity in healthcare professionals' practices, diversity in groups of healthcare professionals included in studies, their professional and workplace characteristics, beliefs, attitudes ${ }^{8}$, and also the result of differences in questions asked. In our study we found a notable difference in the range from around $6 \%$ to $13 \%$ and $14 \%$. They would mostly recommend ECs in case of patients' queries and less likely proactively, as shown in other studies ${ }^{8}$. Other studies have shown that patient interest may be a significant promoter for a healthcare professional's recommendation ${ }^{25}$. When respondents were asked to which groups of patients, if at all, they would recommend ECs, around twothirds chose to none of the patients, while around a third of respondents identified at least one group of the patients to whom they could recommend ECs. This additionally indicates that the decision of the provider may also change when having contact with a specific patient. Other ways of evaluation of counselling practices might be more precise. It is also worth mentioning that respondents would less likely recommend ECs for either purpose proactively than in the case of patients' queries, as shown in other studies ${ }^{8}$. We find similar responses and mostly the same participating healthcare professionals recommend or would recommend ECs for both purposes. The key characteristics of respondents recommending ECs are lower knowledge about ECs, male gender, and ever use of other products (especially EGs). It is also worth mentioning that bivariate analyses show that respondents recommending ECs had high perceived level of knowledge, but low score of actual knowledge, which shows that they might overestimate their own knowledge and recommend ECs based on suboptimal knowledge of actual data and research findings. Lack of knowledge on ECs is an important issue also from another perspective in provider-patient communication. Almost half of respondents were unable to counsel their patients about ECs, the majority, around two-thirds, due to lack of knowledge and one-third due to lack of 
data and research. Lack of knowledge is mostly emphasized by providers working in home nursing and those not providing smoking cessation support, which is not surprising as these respondents are probably those with least training and patient contact regarding smoking cessation among those included in our study. Respondents that emphasize lack of knowledge report low level of perceived and actual knowledge, so they appropriately evaluate their level of knowledge. Other studies already emphasized inability of healthcare professionals to counsel due to lack of knowledge $\mathrm{e}^{8,9,18,19,26}$ or lack of data and research ${ }^{8,9,11,18}$. A substantial proportion, approximately a quarter, of respondents let the patients make the final decision by themselves, even though they would not otherwise recommend ECs. This shows that they somehow tolerate EC use, similar to results of other studies ${ }^{9,19,27}$. Data indicate that this approach, besides not recommending ECs, is most often used by respondents working at Health Education Centres /Health Promotion Centres and others including Quitline counsellors, both at the forefront of smoking cessation in Slovenia, while they have the same level of knowledge as those not recommending ECs. Unfortunately, data do not enable us to find reasons behind this, but maybe the unavailability of clear and enforced workplace guidelines/recommendations on how they should counsel patients regarding ECs could be playing a role. Actually, the vast majority, around $90 \%$, of respondents reports that there are no workplace guidelines/recommendations available or have no knowledge of them being available at their workplace, consistent with some other studies ${ }^{9,21,25,28}$. In our study, respondents from a large minority that reports availability of workplace guidelines/ recommendations are more likely from other provider types (Quitline), other workplaces (Quitline), Health Education Centres/Health Promotion Centres, and those providing smoking cessation support, which could be expected because these are mostly healthcare professionals that receive directions on how to treat ECs at training 'on-the-job', through educational materials and guidelines for their work. Quitline and Health Education Centres/Health Promotion Centres in Slovenia activities are coordinated by National Institute of Public Health (NIPH), so healthcare professionals in these workplaces should be aware of NIPH recommendations. These do not give details on how to work with each specific patient, but clearly state that ECs should not be recommended for smoking cessation or any other purpose. The content of the vast majority of the guidelines/ recommendations, reported by the respondents, is compatible with that given by NIPH to Quitline counsellors and healthcare professionals working at Health Education Centres/Health Promotion Centres, but on the other hand, it is striking that a substantial number of respondent responses from these workplaces indicate that they are not aware of them. Availability of guidelines/recommendations is also more likely reported by those with lower number of years at their current workplace, lower number of years working with patients, and lower number of patients, which indicates that these respondents are newer to their work and have thus probably received more recent training, including about ECs, than other employees.

\section{Strengths and limitations}

Our study is the first in Slovenia to explore counselling practices regarding ECs in healthcare professionals in Slovenia and its results will serve as a guide for future work. The study shows the associations of many different demographic, personal and professional characteristic of respondents with different counselling practices. The study also has several limitations. Calculation of an exact response rate is not possible, because we do not know exactly how many healthcare professionals eventually received the invitation. If calculated for those that received our invitation, then the response rate would be $46.6 \%$, but we do not know to how many of their colleagues the invitation was forwarded. If we take into account all employed in the invited groups of healthcare professionals in Slovenia, and calculate the minimum response rate possible, it represents $24.2 \%$. First, the response rate is not optimal, making nonresponse bias possible and limiting generalisability, but it is comparable to many other studies on a similar topic $^{7,18-23,26,27}$. The study is cross-sectional and allows insight into current perspectives of respondents, which may change with time and evolving research. Also, self-response raises the possibility of social desirability bias. In our study, we used elements in other previous studies, certain questions were newly created and also reliability and validity have not 
been studied. In Slovenia, the number of Quitline counsellors is low, while their response rate was one of the highest among different providers' groups of the study, their absolute number was low $(n=10)$ and as such this group was included among other type of workplace, which might have influenced the results. Majority of respondents were women, but this reflects the actual gender distribution of the studied population. Considerable number of respondents decided to leave the survey before completion and thus missing data were substantial (first questions were answered by 479 respondents, the last about availability of guidelines by 318). Numbers of respondents in some groups were low and did not enable any further analyses.

\section{CONCLUSIONS}

The majority of participating healthcare professionals working in the field of preventive healthcare and smoking cessation in Slovenia do not recommend ECs for smoking cessation or cutting down the number of cigarettes smoked. Knowledge about ECs is the key determinant of recommending them, respondents with lower knowledge on ECs are more likely to recommend them. Lack of knowledge on ECs is an important issue also from another perspective in provider-patient communication as it also disables significantly the responses of participant healthcare professionals in counseling their patients about ECs. Only a significant minority of participating healthcare professionals working in the field of preventive healthcare and smoking cessation in Slovenia reports availability of guidelines/recommendations for counselling patients about ECs at their workplace. This leads to the conclusion that there is a strong need for training and educational materials about ECs and also wide distribution and update of existing recommendations in studied and other groups of healthcare professionals. The focus of these activities should be for all employees, not only those recently employed. It is necessary also to ensure compliance to official guidelines/recommendations, especially in those that are ever or current users of ECs and new tobacco products.

\section{REFERENCES}

1. Bhatnagar A, Payne TJ, Robertson RM. Is There A Role for Electronic Cigarettes in Tobacco Cessation? J Am Heart
Assoc. 2019;8(12):e012742. doi:10.1161/JAHA.119.012742

2. McDonald CF. E-cigarettes for smoking cessation: Current state of play. Respirology. 2019;24(8):722-723. doi:10.1111/resp.13630

3. Bush A, Bhatt J, Grigg J. E cigarettes: Tar Wars: The (Tobacco) Empire Strikes Back. Arch Dis Child. 2018;104(11):10271039. doi:10.1136/archdischild-2018-315820

4. National Academies of Sciences, Engineering, and Medicine. Public health consequences of e-cigarettes. Washington, DC: The National Academies Press; 2018.

5. Hartmann-Boyce J, McRobbie H, Bullen C, Begh R, Stead LF, Hajek P. Electronic cigarettes for smoking cessation. Cochrane Database Syst Rev. 2016;9:CD010216. doi:10.1002/14651858.CD010216.pub3

6. Brown-Johnson CG, Burbank A, Daza EJ, Wassmann A, Chieng A, Rutledge GW, Prochaska JJ. Online PatientProvider E-cigarette Consultations: Perceptions of Safety and Harm. Am J Prev Med. 2016;51(6):882-889. doi:10.1016/j.amepre.2016.06.018

7. Steinberg MB, Giovenco DP, Delnevo CD. Patientphysician communication regarding electronic cigarettes. Prev Med Rep. 2015;2:96-98. doi:10.1016/j.pmedr.2015.01.006

8. Erku DA, Gartner CE, Morphett K, Steadman KJ. Beliefs and self-reported practices of healthcare professionals regarding electronic nicotine delivery systems (ENDS): a mixed-methods systematic review and synthesis. Nicotine Tob Res. 2019:ntz046. doi:10.1093/ntr/ntz046

9. Koprivnikar H, Farkaš Lainščak J. Znanje, stališča, prepričanja in trenutne prakse glede elektronskih cigaret med svetovalci za pomoč pri opuščanju kajenja in zdravstvenimi delavci [Knowledge, attitudes, beliefs and current practices related to electronic cigarettes among smoking cessation advisors and healthcare professionals]. ANALI PAZU. 2018;8(1-2):40-49. http:// anali-pazu.si/sites/default/files/farka \% C5\%A1\%20 lain\%C5\%A1\%C4\%8Dak_jerneja.pdf. Accessed September $26,2019$.

10. Livingston CJ, Freeman RJ, Costales VC, Westhoff JL, Caplan LS, Sherin KM, Niebuhr DW. Electronic Nicotine Delivery Systems or E-cigarettes: American College of Preventive Medicine's Practice Statement. Am J Prev Med. 2019;56(1):167-178. doi:10.1016/j.amepre.2018.09.010

11. Bhatnagar A, Whitsel LP, Blaha MJ, et al. New and Emerging Tobacco Products and the Nicotine Endgame: The Role of Robust Regulation and Comprehensive Tobacco Control and Prevention: A Presidential Advisory from the American Heart Association. Circulation. 2019;139(19):e937-e958. doi:10.1161/CIR.0000000000000669

12. Bals R, Boyd J, Esposito S, et al. Electronic cigarettes: a task force report from the European Respiratory Society. Eur Respir J. 2019;53(2). doi:10.1183/13993003.01151-2018

13. Barua RS, Rigotti NA, Benowitz NL, et al. 2018 ACC Expert Consensus Decision Pathway on Tobacco Cessation Treatment: A Report of the American College 
of Cardiology Task Force on Clinical Expert Consensus Documents. J Am Coll Cardiol. 2018;72(25):3332-3365. doi:10.1016/j.jacc.2018.10.027

14. Brady BR, De La Rosa JS, Nair US, Leischow SJ. Electronic Cigarette Policy Recommendations: A Scoping Review. Am J Health Behav. 2019;43(1):88-104. doi:10.5993/AJHB.43.1.8

15. Koprivnikar H, Stepan D, Kukec A, Farkaš Lainščak J. Uporaba elektronskih cigaret med študenti Medicinske in Zdravstvene fakultete Univerze v Ljubljani. Medicinski razgledi. 2018;57(2):169-186.

16. Koprivnikar H. Kajenje. Kako skrbimo za zdravje? In: Z zdravjem povezan vedenjski slog prebivalcev Slovenije, 2016. Ljubljana: Nacionalni inštitut za javno zdravje; 2018.

17. Singh B, Hrywna M, Wackowski OA, Delnevo CD, Jane Lewis M, Steinberg MB. Knowledge, recommendation, and beliefs of e-cigarettes among physicians involved in tobacco cessation: A qualitative study. Prev Med Rep. 2017;8:25-29. doi:10.1016/j.pmedr.2017.07.012

18. Kanchustambham V, Saladi S, Rodrigues J, Fernandes H, Patolia S, Santosh S. The knowledge, concerns and healthcare practices among physicians regarding electronic cigarettes. J Community Hosp Intern Med Perspect. 2017;7(3):144-150. doi:10.1080/20009666.2017.1343076

19. Nickels AS, Warner DO, Jenkins SM, Tilburt J, Hays JT. Beliefs, Practices, and Self-efficacy of US Physicians Regarding Smoking Cessation and Electronic Cigarettes: A National Survey. Nicotine Tob Res. 2017;19(2):197207. doi:10.1093/ntr/ntw194

20. Hiscock R, Bauld L, Arnott D, Dockrell M, Ross L, McEwen A. Views from the Coalface: What Do English Stop Smoking Service Personnel Think about E-Cigarettes? Int J Environ Res Public Health. 2015;12(12):16157-16167. doi:10.3390/ijerph121215048

21. Sherratt FC, Newson L, Field JK. Electronic cigarettes: a survey of perceived patient use and attitudes among members of the British thoracic oncology group. Respir Res. 2016;17(1):55. doi:10.1186/s12931-016-0367-y

22. Shin DW, Kim YI, Kim SJ, et al. Lung cancer specialist physicians' attitudes towards e-cigarettes: A nationwide survey. PLoS One. 2017;12(2):e0172568. doi:10.1371/journal.pone.0172568

23. Pepper JK, Gilkey MB, Brewer NT. Physicians' Counseling of Adolescents Regarding E-Cigarette Use. J Adolesc Health. 2015;57(6):580-586. doi:10.1016/j.jadohealth.2015.06.017

24. Kandra KL, Ranney LM, Lee JG, Goldstein AO. Physicians' attitudes and use of e-cigarettes as cessation devices, North Carolina, 2013. PLoS One. 2014;9(7):e103462. doi:10.1371/journal.pone.0103462

25. El-Shahawy O, Brown R, ElstonLafata J. Primary Care Physicians' Beliefs and Practices Regarding E-Cigarette Use by Patients Who Smoke: A Qualitative Assessment. Int J Environ Res Public Health. 2016;13(5):445. doi:10.3390/ijerph13050445

26. Egnot E, Jordan K, Elliott JO. Associations with resident physicians' early adoption of electronic cigarettes for smoking cessation. Postgrad Med J. 2017;93(1100):319325. doi:10.1136/postgradmedj-2016-134058

27. Van Gucht D, Baeyens F. Health professionals in Flanders perceive the potential health risks of vaping as lower than those of smoking but do not recommend using e-cigarettes to their smoking patients. Harm Reduct J. 2016;13(1):22. doi:10.1186/s12954-016-0111-4

28. Bascombe TM, Scott KN, Ballard D, Smith SA, Thompson W, Berg CJ. Primary healthcare provider knowledge, beliefs and clinic-based practices regarding alternative tobacco products and marijuana: a qualitative study. Health Educ Res. 2016;31(3):375-83. doi:10.1093/her/cyv103

\section{ACKNOWLEDGEMENTS}

We thank Monika Azman, president of Nurses and Midwives Association of Slovenia, Andreja Krajnc, president of Professional group of nurses and health technicians in community care at Nurses and Midwives Association of Slovenia, Sanja Vrbovsek, National Coordinator for Health Education, Tadeja Hocevar, National Coordinator of Quitline telephone in 2018, Karmen Henigsman and Sanja Krusic, all from National Institute of Public Health for helping with inviting healthcare professionals from respective groups to participate in the study. We also thank Borut Jug for his assistance in obtaining data from healthcare professionals working in Outpatient Cardiac Rehabilitation Units and Antonija Poplas Susic for her advice regarding possible approaches to include healthcare professionals from Family Medicine Practices in the study.

\section{CONFLICTS OF INTEREST}

The authors have completed and submitted the ICMJE Form for Disclosure of Potential Conflicts of Interest and none was reported.

\section{FUNDING}

The authors acknowledge the project 'Assessment of attitudes, knowledge and current practices related to electronic cigarettes among healthcare professionals working in the field of preventive healthcare and smoking cessation, with preparation of educational material for unified and evidence-based counselling to patients' (ID V3-1729) which was financially supported by the Slovenian Research Agency and Ministry of Health of the Republic of Slovenia.

\section{AUTHORS' CONTRIBUTIONS}

All three authors contributed to the design and implementation of the study, to the analysis of the results and to the writing of the manuscript.

\section{PROVENANCE AND PEER REVIEW}

Not commissioned; externally peer reviewed. 